\title{
Sustainable defect repair
}

The lifetimes of organic-inorganic solar cells are considerably shorter than silicon-based and other inorganic-based solar cells, which hampers their commercialization. In lead-iodide perovskite solar cells, these lifetimes are reduced when a steep reduction is observed in the power conversion efficiency (PCE) of the device, in part because of the intrinsic degradation of perovskite absorbers. One type of this intrinsic degradation, to form $\mathrm{Pb}^{0}$ and $\mathrm{I}^{0}$ defects, occurs in typical working conditions as a consequence of electrical fields, light and heat. These $\mathrm{Pb}^{0}$ and $\mathrm{I}^{0}$ defects may act as recombination centres for carriers and initiate detrimental chemical reactions.

Now, writing in Science, Huanping Zhou, Ling-Dong Sun, Chun-Hua Yan and colleagues report a sustainable redox process for recovering $\mathrm{Pb}^{0}$ and $\mathrm{I}^{0}$ defects within a pervoskite absorber and hence enhance the durability of devices under various aging conditions. The simultaneous reactions involving $\mathrm{Pb}^{0}$ and $\mathrm{I}^{0}$ defects are achieved by the incorporation of a europium ion pair $\left(\mathrm{Eu}^{3+}-\mathrm{Eu}^{2+}\right)$ within the perovskite absorber. More specifically, the trivalent $\mathrm{Eu}^{3+}$ oxidizes $\mathrm{Pb}^{0}$ to $\mathrm{Pb}^{2+}$, and the divalent $\mathrm{Eu}^{2+}$, which is formed in the oxidation step, reduces $\mathrm{I}^{0}$ to $\mathrm{I}^{-}$. This transfer, or shuttling, of electrons is a cyclic process and each ion of the ion pair is replenished. As a result of the nonvolatility of the ion pair, the defects are continually eliminated and hence this 'redox shuttle' is more effective than using an additive that is specific for only one defect and consumed over time.

"The elimination of the $\mathrm{Pb}^{0}$ and $\mathrm{I}^{0}$ defects within the perovskite absorber of the solar cells achieves substantially improved PCE and long-term durability," says Zhou. The researchers compared the performances of $\mathrm{Eu}^{3+}$ incorporated devices and reference devices, as well as probed the influence of light and thermal stresses on their performances. After 1,000 hours of continuous illumination or heating at $85^{\circ} \mathrm{C}$, the $\mathrm{Eu}^{3+}$-incorporated half devices retained $93 \%$ and $91 \%$, respectively, of the initial PCE, whereas the reference devices retained $78 \%$ and $67 \%$, respectively. "By modification of

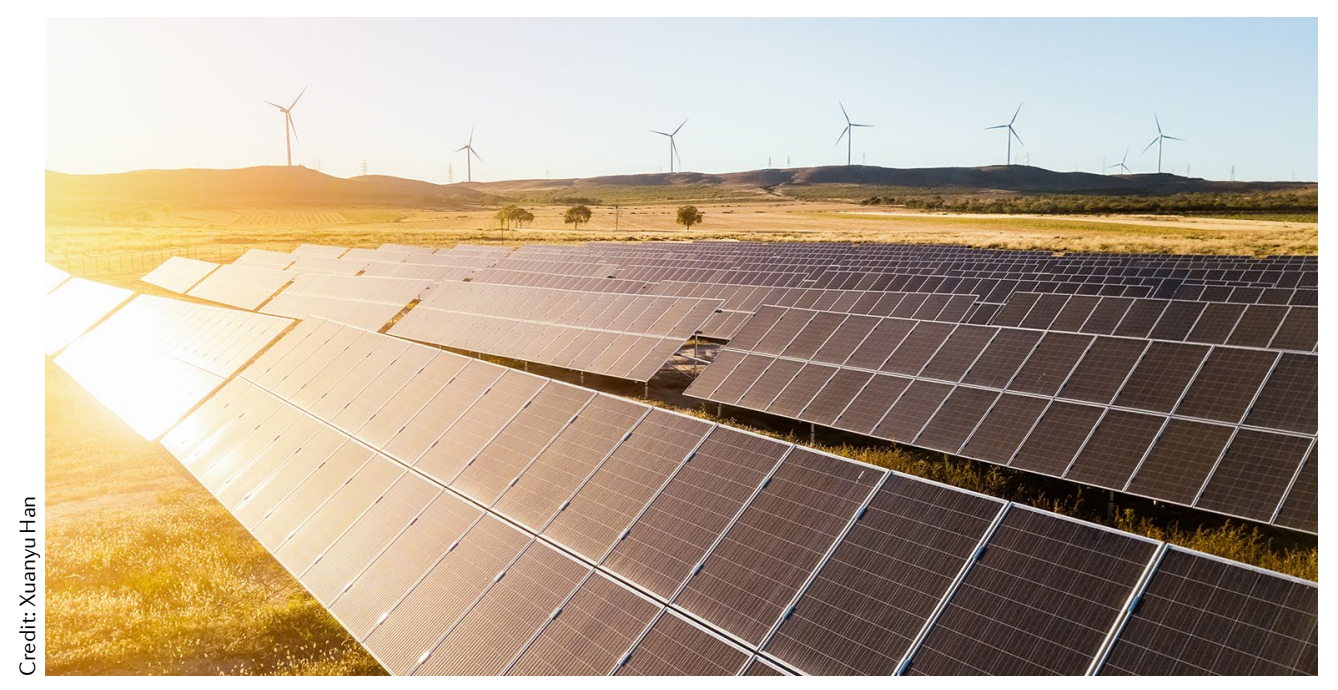

the hole-transport materials with a conductive polymer, the $\mathrm{Eu}^{3+}$ incorporated full devices maintained $92 \%$ and $89 \%$ of their initial PCE under the same light or thermal stress, respectively, for 1,500 hours," explains Zhou. "By contrast, under the same conditions, the reference devices maintained only $51 \%$ and $32 \%$, respectively."

The shelf time of the solar cells was probed following storage in an inert environment. "The $\mathrm{Eu}^{3+}-\mathrm{Eu}^{2+}$ redox-shuttle devices maintained $90 \%$ of their initial PCE even after 8,000 hours because of improved long-term open-circuit voltage, short-circuit current density and fill factor stability," says Zhou. "While the reference devices could only maintain $10 \%$ of the initial PCE."

The researchers screened a variety of additives, however, $\mathrm{Eu}^{3+}-\mathrm{Eu}^{2+}$ was identified as the most effective redox shuttle as a result of the non-volatility and the practicable redox potential of the ion pair. However, they envisage investigating a range of additives to identify if degradation problems in other inorganic semiconductor absorbers may be solved in a similar way.

In the future, the researchers aim to probe the kinetics of the redox shuttle process. To investigate the kinetics, both the speed and mechanism of the intrinsic degradation within the perovskite materials must be better understood. "In addition, we will focus on the identification of other instability issues and explore the solutions for these issues to enhance stability and hence promote the commercialization of perovskite solar cell technology," concludes Zhou.

Alison Stoddart

ORIGINAL ARTICLE Wang, L. et al. A Eu ${ }^{3+}-\mathrm{Eu}^{2+}$ ion redox shuttle imparts operational durability to $\mathrm{Pb}-\mathrm{I}$ perovskite solar cells. Science 363, 265-270 (2019) 